\title{
Tumor proteomic profiling predicts the susceptibility of breast cancer to chemotherapy
}

\author{
JIANBO HE ${ }^{1,2}$, DEJUN SHEN ${ }^{1,2,5}$, DEBRA U. CHUNG ${ }^{2,3}$, ROMAINE E. SAXTON ${ }^{1,2}$, \\ JULIAN P. WHITELEGGE ${ }^{4}$, KYM F. FAULL ${ }^{4}$ and HELENA R. CHANG ${ }^{1,2,3}$ \\ ${ }^{1}$ Gonda/UCLA Breast Cancer Research Laboratory, ${ }^{2}$ Department of Surgery, ${ }^{3}$ Revlon/UCLA Breast Center, ${ }^{4}$ Pasarow Mass \\ Spectrometry Laboratory, Semel Institute and Department of Psychiatry \& Biobehavioral Science, David Geffen School \\ of Medicine, University of California at Los Angeles, Los Angeles, CA 90095; ${ }^{5}$ Department of Pathology, \\ Keck School of Medicine, University of Southern California, Los Angeles, CA 90033, USA
}

Received March 20, 2009; Accepted May 26, 2009

DOI: 10.3892/ijo_00000380

\begin{abstract}
Chemotherapy is often used for breast cancer treatment, but individual outcome varies widely. We hypothesized that tumor proteomic profiles obtained prior to chemotherapy may predict the individual tumor response to treatment. The goal of our study was to explore feasibility of using proteomic profiling to preselect patients for an effective chemotherapeutic regimen. Tumors from 52 patients with T2-T4 breast cancer were prospectively collected before neoadjuvant chemotherapy, and were analyzed using surface-enhanced laser desorption ionization/time of flight (SELDI) mass spectrometry. Mass spectral profiles were obtained from tumors with various sensitivities to chemotherapy. Both nonsupervised hierarchical clustering and supervised neural
\end{abstract}

Correspondence to: Dr Helena R. Chang, Revlon/UCLA Breast Center, Department of Surgery, UCLA David Geffen School of Medicine, 200 Medical Plaza, B265, Los Angeles, CA 90095, USA E-mail: hchang@mednet.ucla.edu

Abbreviations: ANOVA, analysis of variance; AUC, area under the curve; DCIS, ductal carcinoma in situ; DLDA, diagonal linear discriminant analysis; FDR, false discovery rate; FISH, fluorescence in situ hybridization; GEPAS, gene expression pattern analysis suite; IR, intermediate responders $(>25 \%$ but $\leq 75 \%$ tumor regression); KNN, K nearest neighbor; MALDI-TOF, matrix-assisted laser desorption ionization/ time of flight; MS, mass spectrometry; NR, non-responders, chemo-resistant tumors ( $\leq 25 \%$ tumor regression); PAM, prediction analysis with microarrays; $\mathrm{pCR}$, pathological complete response, no residual cancer found at primary tumor site; $\mathrm{R}$, responders ( $>75 \%$ of tumor regression); ROC, receiver operator characteristic; SELDI-TOF, surface-enhanced laser desorption ionization/time of flight; SOM, self-organizing map; ST, chemo-susceptible tumors ( $>25 \%$ tumor regression); SVM, support vector machines; T2, primary tumor size more than $2 \mathrm{~cm}$ and up to $5 \mathrm{~cm}$; T3, primary tumor size more than $5 \mathrm{~cm}$; T4, primary tumor of any size with direct invasion to chest wall or skin; TIC, total ion current; TRR, tumor regression rate

Key words: proteomic biomarker, breast cancer, neoadjuvant chemotherapy, mass spectrometry, bioinformatics network-based classification approaches were employed to compare the profiles. The first two thirds of the enrolled cases (35) were allocated to a training set to select peaks characteristic of resistant tumors. The candidate peaks were used to develop a predicting rule to evaluate the remaining 17 specimens in the validation set. In the training set, the most prominent differences were found between drug resistant and drug susceptible tumors by non-supervised hierarchical clustering. In the validation set, the supervised classification with the $\mathrm{K}$ nearest neighbor (KNN) model correctly classified most tumor responses with an accuracy rate of $92.3 \%$ [100\% of resistant tumors (4/4), and $84.6 \%$ of the tumors with favorable response (11/13)]. In the entire group, a single peak at $\mathrm{m} / \mathrm{z} 16,906$ correctly separated $88.9 \%$ of the tumors with pathologically complete response, and $91.7 \%$ of the resistant tumors. The data suggest that breast cancer protein biomarkers may be used to pre-select patients for optimal chemotherapeutic treatment.

\section{Introduction}

Preoperative chemotherapy (neoadjuvant chemotherapy) has gained wide acceptance for treating patients with locally advanced breast cancer. In these patients the most acknowledged benefit is the reduction in tumor size allowing either a complete resection of an otherwise unresectable tumor or breast conservation surgery in some patients with large tumors. Furthermore, tumor response to neoadjuvant treatment has been shown to be prognostically significant. Other less proven benefits of neoadjuvant chemotherapy include eradication of microscopic metastases and improved survivals (1-3). Postoperative adjuvant chemotherapy has been used successfully to improve survival rates in patients with early stage breast cancer (4). Although many patients benefit from chemotherapy, some fail to respond. However, the success of chemotherapy, whether pre- or post-operative, in any given individual cannot be predicted. The uncertain benefit for a particular individual, as well as significant toxicity of chemotherapy in all patients, calls for development of methods to select the right patients for treatment and spare those who will not benefit. 
There already is precedence for using the expression of specific proteins by tumors to select treatment and to monitor disease progression. For example, estrogen and progesterone receptor levels in tumor tissues are now used to predict benefit of Tamoxifen therapy in women of all ages (5), ovarian ablation in premenopausal patients $(6,7)$ and aromatase inhibitor treatment in postmenopausal women $(5,8,9)$. More recently HER $2 /$ neu status also has been used to predict effectiveness of the combination of chemotherapy and Herceptin in women with HER2/neu positive breast cancer (10-12). The Breast Cancer International Research Group conducted a multicenter phase III trial to investigate the best combination of chemotherapy and Herceptin treatment for these patients. The study concluded that Herceptin with chemotherapy was superior to chemotherapy alone in patients with HER2 positive early-stage breast cancer (Slamon et al, 29th Annual San Antonio Breast Cancer Symposium, abs. 52, 2006). Estrogen and progesterone receptor levels and HER2/ neu status also were prognostically valuable in these patients.

However, no reliable biomarkers currently are available to predict the response of an individual to a particular type of chemotherapy. Several recent studies have attempted to use gene expression profiling and bioinformatics to predict the need for adjuvant chemotherapy in patients with early stage breast cancer (13-15). The Oncotype DX Breast Cancer Assay uses reverse transcriptase-polymerase chain reaction to correlate the expression of a panel of 21 genes in hormone receptor positive and lymph node negative tumors to predict the risk of disease recurrence. A score calculated from the expression of these genes predicts the risk of tumor recurrence after initial Tamoxifen treatment, and is used to assess the need for further chemotherapy. This study showed that the difference in the risks of distant recurrence between patients with low and high scores by the Oncotype DX test was statistically significant and clinically useful (14).

Similar to the genomic analysis of cancer-related genes, proteomics has emerged as a potentially powerful tool for tumor classification through protein biomarker profiling. It is now widely recognized that proteomics has the potential to improve disease diagnosis and management (16). However, the proteome changes constantly due to the influence of many factors such as aging, disease and environmental exposure. Thus, studying the cellular or circulating proteome has been more challenging than the genome (17). The application of proteomics to monitor tumor response to chemotherapy is only in the early stages, and our current study was specifically designed to compare SELDI mass spectra of breast cancer tissues from chemotherapy responsive and non-responsive patients receiving the same neoadjuvant treatment.

Evaluation of chemotherapy response in these patients is measured both by microscopic examination of surgically removed tissue (pathological examination), and by physical examination and/or imaging studies (clinical examination). The pathological response is usually classified as either pathologically complete response (pCR) or having residual tumor after neoadjuvant treatment. Clinical responses are graded as a change in tumor size from baseline to postneoadjuvant treatment: complete response (no tumor detected), partial response (tumor shrinks $\geq 50 \%$ ), marginal response (tumor shrinks 25-49\%), stable disease (tumor size changes
$<25 \%$ ), and tumor progression (tumor size increases $>25 \%$ ). The latter two groups are frequently considered non-responders. It was our goal to use proteomic technologies to study differences between drug resistant and sensitive tumors in an effort to improve tumor classification and patient selection for individualized chemotherapy.

\section{Materials and methods}

Patients and clinical information. Fresh tumor tissues from 52 patients with non-metastatic T2-T4 breast cancer were prospectively collected for this study. All patients participated in an Institutional Review Board (IRB) approved phase II clinical trial to study the efficacy of neoadjuvant Taxotere/ Carboplatin \pm Herceptin therapy. The patient inclusion criteria for the clinical trial were: (i) females aged 18 and older with a tissue-proven diagnosis of stage II-III invasive breast cancer; (ii) measurable primary breast cancer; (iii) no prior chemotherapy for the current breast cancer or prior radiation of the involved breast; (iv) available tissue estrogen and progesterone receptor status by immunohistochemical staining, and HER2/neu status by the fluorescent in situ hybridization (FISH) test; and (v) completion of the clinical staging requirements. The patient exclusion criteria included: (i) refusal of definitive resection of primary tumor; (ii) evidence of metastasis; (iii) pregnancy or nursing conditions, and fertile women not on birth control. Written informed consent was obtained from all participants and baseline clinical assessment of tumor size, tissue diagnosis and tumor biomarker status was recorded.

Because a baseline tumor size by pathologic evaluation was not possible, the clinically measured tumor size prior to chemotherapy was used as baseline. Post-chemotherapy information including tumor size measured by both clinical and pathological methods, lymph node staging, and tumor biomarkers were collected from each definitive breast cancer surgery. The formula used to calculate tumor regression rate (TRR) was as follows: (baseline tumor size-residual tumor size)/baseline tumor size, where the baseline tumor size was measured clinically and the post chemotherapy residual invasive tumor size was measured pathologically. The TRR was categorized into three groups: (i) responders (TRR $>75 \%$, $\mathrm{R})$, (ii) intermediate responders ( $25 \%<\mathrm{TRR} \leq 75 \%$, IR) and (iii) non-responders with minimal to no response or tumor progression (TRR $\leq 25 \%, \mathrm{NR}$ ).

Tumor tissue collection and sample preparation. Breast cancer tissues were prospectively collected from each patient at two different time points: (i) at baseline before chemotherapy and (ii) at the time of definitive cancer surgery after completion of neoadjuvant chemotherapy for proposed proteomic study. Tumor specimens were placed on ice immediately after removal, and transferred to the UCLA Breast Cancer Tissue Bank. When specimens were obtained from sites other than UCLA, they were delivered on ice and received for processing within $24 \mathrm{~h}$ of sample collection. Upon arrival, the specimens were grossly dissected to remove excess adipose tissue before dividing into three parts and storage at $-80^{\circ} \mathrm{C}$.

In the results reported here, tissue specimens collected at baseline before chemotherapy were extracted by homogenizing 
Table I. Patient characteristics.

\begin{tabular}{|c|c|c|c|}
\hline Characteristics & Total patients (\%) & Training set (\%) & Validation set $(\%)$ \\
\hline \multicolumn{4}{|l|}{ Age, years } \\
\hline$\geq 50$ & $26(50.0)$ & $18(51.4)$ & $8(47.1)$ \\
\hline$<50$ & $26(50.0)$ & $17(48.6)$ & $9(52.9)$ \\
\hline Mean age & 49.4 & 49.3 & 49.7 \\
\hline \multicolumn{4}{|l|}{ Tumor histology ${ }^{a}$} \\
\hline IDC & $42(80.8)$ & $27(77.1)$ & $15(88.2)$ \\
\hline ILC & $8(15.4)$ & $7(20.0)$ & $1(5.9)$ \\
\hline IDC+ILC & $1(1.9)$ & 0 & $1(5.9)$ \\
\hline ITC+IDC & $1(1.9)$ & $1(2.9)$ & 0 \\
\hline \multicolumn{4}{|l|}{ Tumor stage } \\
\hline $\mathrm{T} 2$ & $5(9.6)$ & 0 & $5(29.4)$ \\
\hline $\mathrm{T} 3$ & $32(61.5)$ & $26(74.3)$ & $6(35.3)$ \\
\hline $\mathrm{T} 4$ & $15(28.8)$ & $9(25.7)$ & $6(35.3)$ \\
\hline \multicolumn{4}{|l|}{ Tumor differentiation } \\
\hline Poor & $13(25)$ & $8(22.9)$ & $5(29.4)$ \\
\hline Moderate & $7(13.5)$ & $6(17.2)$ & $1(5.9)$ \\
\hline Well & $6(11.5)$ & $3(8.6)$ & $3(17.6)$ \\
\hline Unknown & $26(50)$ & $18(51.4)$ & $8(47.1)$ \\
\hline \multicolumn{4}{|l|}{ Tumor grade } \\
\hline High & $16(30.8)$ & $11(31.4)$ & $5(29.4)$ \\
\hline Intermediate & $11(21.2)$ & $7(20)$ & $4(23.5)$ \\
\hline Low & $6(11.5)$ & $5(14.3)$ & $1(5.9)$ \\
\hline Unknown & $19(36.5)$ & $12(34.3)$ & $7(41.2)$ \\
\hline \multicolumn{4}{|l|}{ Tumor IHC marker ${ }^{\mathrm{b}}$} \\
\hline ER positive & $33(63.5)$ & $21(60.0)$ & $12(70.6)$ \\
\hline PR positive & $21(40.4)$ & $14(40.0)$ & $7(41.2)$ \\
\hline HER2/neu positive & $21(40.4)$ & $15(42.9)$ & $6(35.3)$ \\
\hline \multicolumn{4}{|l|}{ Neoadjuvant chemotherapy ${ }^{\mathrm{c}}$} \\
\hline $\mathrm{TC}$ & $31(59.6)$ & $20(57.1)$ & $11(64.7)$ \\
\hline $\mathrm{TCH}$ & $10(19.2)$ & $8(22.9)$ & $2(11.8)$ \\
\hline $\mathrm{TC} / \mathrm{H}$ & $11(21.2)$ & $7(20.0)$ & $4(23.5)$ \\
\hline \multicolumn{4}{|l|}{ Ethnicity } \\
\hline Asian & $8(15.4)$ & $5(14.3)$ & $3(17.6)$ \\
\hline Black & $4(7.7)$ & $2(5.7)$ & $2(11.8)$ \\
\hline Hispanic & $8(15.4)$ & $7(20.0)$ & $1(5.9)$ \\
\hline White & $32(61.5)$ & $21(60.0)$ & $11(64.7)$ \\
\hline \multicolumn{4}{|l|}{ Surgery $^{\mathrm{d}}$} \\
\hline Mastectomy & $33(63.5)$ & $22(62.9)$ & $11(64.7)$ \\
\hline Breast-conserving treatment & $19(36.5)$ & $13(37.1)$ & $6(35.3)$ \\
\hline
\end{tabular}

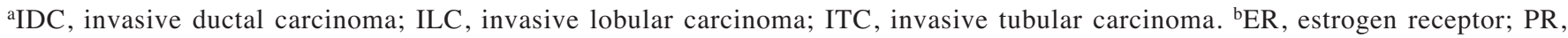
progesterone receptor. ${ }^{\mathrm{c}} \mathrm{TC}$, preoperative Taxotere $75 \mathrm{mg} / \mathrm{m}^{2}$, carboplatin (AUC=6) every three weeks for four cycles; TCH, preoperative TC with weekly Herceptin at a loading dose of $4 \mathrm{mg} / \mathrm{kg}$ and subsequent dose of $2 \mathrm{mg} / \mathrm{kg}$; TC/H, TC preoperatively with Herceptin given after surgery. ${ }^{\mathrm{d}}$ Mastectomy includes modified radical mastectomy (MRM) and simple mastectomy (SM).

the frozen tissue in liquid nitrogen, followed by suspension in $1 \%$ Triton $\mathrm{X}-100$. The samples were re-frozen at $-80^{\circ} \mathrm{C}$ and then thawed on ice for $30 \mathrm{~min}$. The freeze/thaw process was repeated, and each suspension was subjected to centrifugation $\left(10,000 \mathrm{x} \mathrm{g}, 10 \mathrm{~min}, 4^{\circ} \mathrm{C}\right)$. The supernatants were then subjected to albumin and immunoglobulin depletion using an albumin and $\mathrm{IgG}$ removal kit (Amersham) as well as hemoglobin depletion using Ni-NTA magnetic agarose beads (Qiagen). Briefly, $20 \mu \mathrm{l}$ of the tissue homogenate supernatant was added to a $320 \mu 1$ slurry containing the antibody-coated beads to first remove $\operatorname{IgG}$ and albumin. The mixture was first incubated at room temperature for $30 \mathrm{~min}$ with gentle rotation, then transferred to a microspin column, and centrifuged $(10,000 \mathrm{x} \mathrm{g}, 5 \mathrm{~min}$, room temperature). Forty microliters of the 


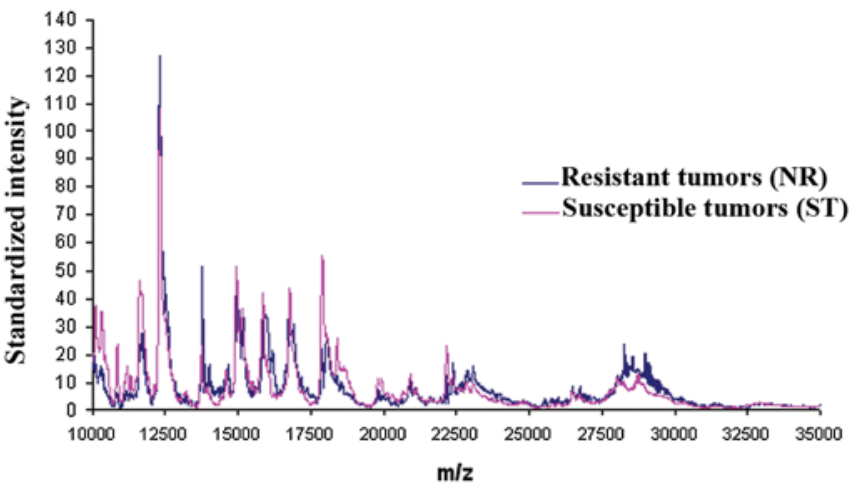

Figure 1. Normalized median intensity SELDI-TOF mass spectra of resistant (NR) and susceptible (ST) tumors. SELDI-TOF spectra of breast cancer tissue extracts from the chemotherapy resistant $(\mathrm{NR}$, blue; $\mathrm{n}=12)$ and susceptible (ST, pink; $n=40$ ) groups.

filtrate was then added to $20 \mu \mathrm{l}$ of Ni-NTA magnetic agarose beads and the mixture was incubated with gentle rotation. A magnetic particle concentrator (Dynal) was used to remove the beads. Two microliters of the final supernatant was mixed with $2 \mu \mathrm{l}$ of freshly prepared sinapinic acid solution $(20 \mathrm{mg} / \mathrm{ml}$ in water/acetonitrile/trifluoroacetic acid, 30/70/0.1, v/v/v), and $2 \mu \mathrm{l}$ of the mixture was loaded in duplicate onto NP20 ProteinChips (Ciphergen Biosystems Inc.).

Surface enhanced laser desorption ionization mass spectrometry (SELDI-MS). Linear time-of-flight mass spectra were recorded without time-lagged source focusing (Model PBS II, Ciphergen Biosystems Inc.) using external calibration with bovine cytochrome C (12,230.9 Da), B-lactoglobin A $(18,363.3 \mathrm{Da})$, bovine serum albumin $(66,410.0 \mathrm{Da})$ and $\mathrm{IgG}$ $(147,300.0 \mathrm{Da})$. Data acquisitions were made using the instrument-supplied automated protocol with the following settings: high mass for acquisition, $100 \mathrm{kDa}$; optimization range $10-100 \mathrm{kDa}$; laser intensity 250 arbitrary Units; detector sensitivity 9 . The raw data were collected using the instrumentsupplied software (Ciphergen ProteinChip ${ }^{\circledR}$ Software 3.1).

Normalization of the mass spectra and peak alignment. After baseline subtraction, the spectra were normalized by dividing the raw signal intensities by the area under the curve (AUC) between $\mathrm{m} / \mathrm{z} 10,000$ and 35,000. Data outside of this range were omitted from each profile. The intensities of signals beyond $\mathrm{m} / \mathrm{z} 35,000$ were close to zero, and the signal intensities below $\mathrm{m} / \mathrm{z} 10,000$ were unusable because in most cases individual peaks could not be distinguished in this range. This normalization process is equivalent to total ion current (TIC) normalization since the number of observations between $\mathrm{m} / \mathrm{z}$ $10 \mathrm{~K}$ and $35 \mathrm{~K}$ were about the same in all samples (data not shown).

Peak alignment/clustering was carried out using the maximal clique method of $\mathrm{Li}$ and Gentleman (18) using the within duplicate error equation to form the boundaries around each $\mathrm{m} / \mathrm{z}$ signal. Analysis of $\mathrm{m} / \mathrm{z}$ error showed that the error was a linear function of $\mathrm{m} / \mathrm{z}$ of the form duplicate $\mathrm{m} / \mathrm{z}$ error $=$ $\mathrm{e}=\mathrm{a}+\mathrm{b}$ average $\mathrm{m} / \mathrm{z}$. For a given signal, the $\mathrm{m} / \mathrm{z}$ bounds were defined as $[\mathrm{m} / \mathrm{z}-\mathrm{e}, \mathrm{m} / \mathrm{z}+\mathrm{e}]$. The $\mathrm{i}^{\text {th }}$ cluster ( $\mathrm{i}^{\text {th }}$ 'clique') was defined as the union of all signals whose $\mathrm{m} / \mathrm{z}$ bounds

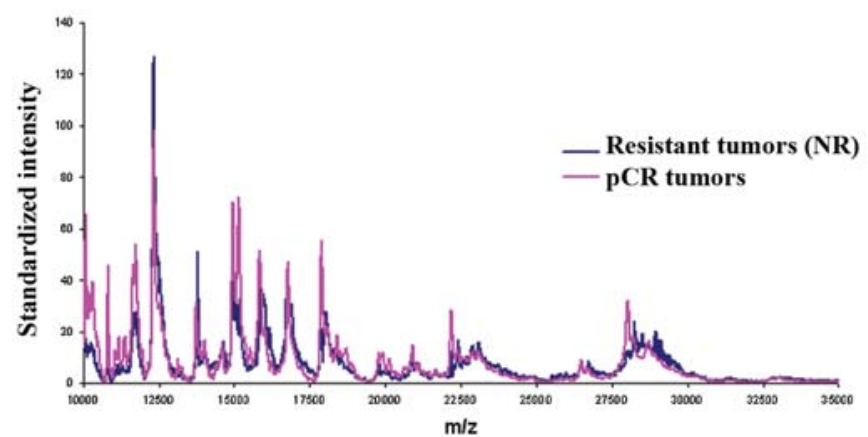

Figure 2. Normalized median intensity SELDI-TOF mass spectra of resistant (NR) and pathologically complete response (pCR) tumors. SELDI-TOF spectra of breast cancer tissue extracts from the chemotherapy resistant (NR, blue; $n=12$ ) and pathologically complete response ( $\mathrm{pCR}$, pink; $n=9)$ tumors.

overlap with the bounds of any other signal. Signals in the same cluster were assumed to have the same true $\mathrm{m} / \mathrm{z}$, and were aligned by assigning the peak the average $\mathrm{m} / \mathrm{z}$ value of all the signals in the cluster. If a sample did not have a peak in a given cluster, the non-peak normalized intensity value at the cluster mean $\mathrm{m} / \mathrm{z}$ was used.

Univariate analysis/comparison. The distribution of the aligned and normalized peak intensities were compared between any two patient groups at each $\mathrm{m} / \mathrm{z}$ signal using the non-parametric Wilcoxon rank sum test. Comparison among the three patient groups (NR, IR and R) at each $\mathrm{m} / \mathrm{z}$ signal was made using the parametric $\mathrm{F}$ statistic. In addition, a receiver operator characteristic (ROC) analysis was carried out for each $\mathrm{m} / \mathrm{z}$ signal. In the ROC analysis, a candidate threshold value was chosen and the percent of intensities less than the threshold in each group was computed. The threshold was varied until the value of the threshold that maximizes the difference in the percentage between the two groups was found.

Multivariate bioinformatic analysis. Multivariate bioinformatic analysis of the normalized and aligned spectral peaks was performed using Gene Expression Pattern Analysis Suite v4.0 (GEPAS, http://gepas.bioinfo.cipf.es), a web-based microarray software package. The cohort was arbitrarily divided into two groups: the first two thirds of the enrolled patients (35 cases) were allocated to a training set to select the $\mathrm{m} / \mathrm{z}$ peaks that appeared to be characteristic of tumor susceptibility to chemotherapy. The remaining 17 cases were assigned to the validation set to confirm the classification of each tumor by the candidate $\mathrm{m} / \mathrm{z}$ signals.

First, the 35 cases in the training set were divided into the $\mathrm{NR}$, IR and $\mathrm{R}$ groups according to the residual tumors found after chemotherapy. An analysis of variance (ANOVA) test was used to compare the spectral differences at each peak location among the three groups with a liberal significance criterion $\mathrm{p}<0.25$ (FDR-indep). The preprocessed data were then used to select significant spectral peaks differentiating the groups. According to the bioinformatic analysis criteria used in our two previous studies $(19,20)$, we selected both the fold difference of at least 2 and statistical significance $<0.05$ to define significant $\mathrm{m} / \mathrm{z}$ peak differences. 


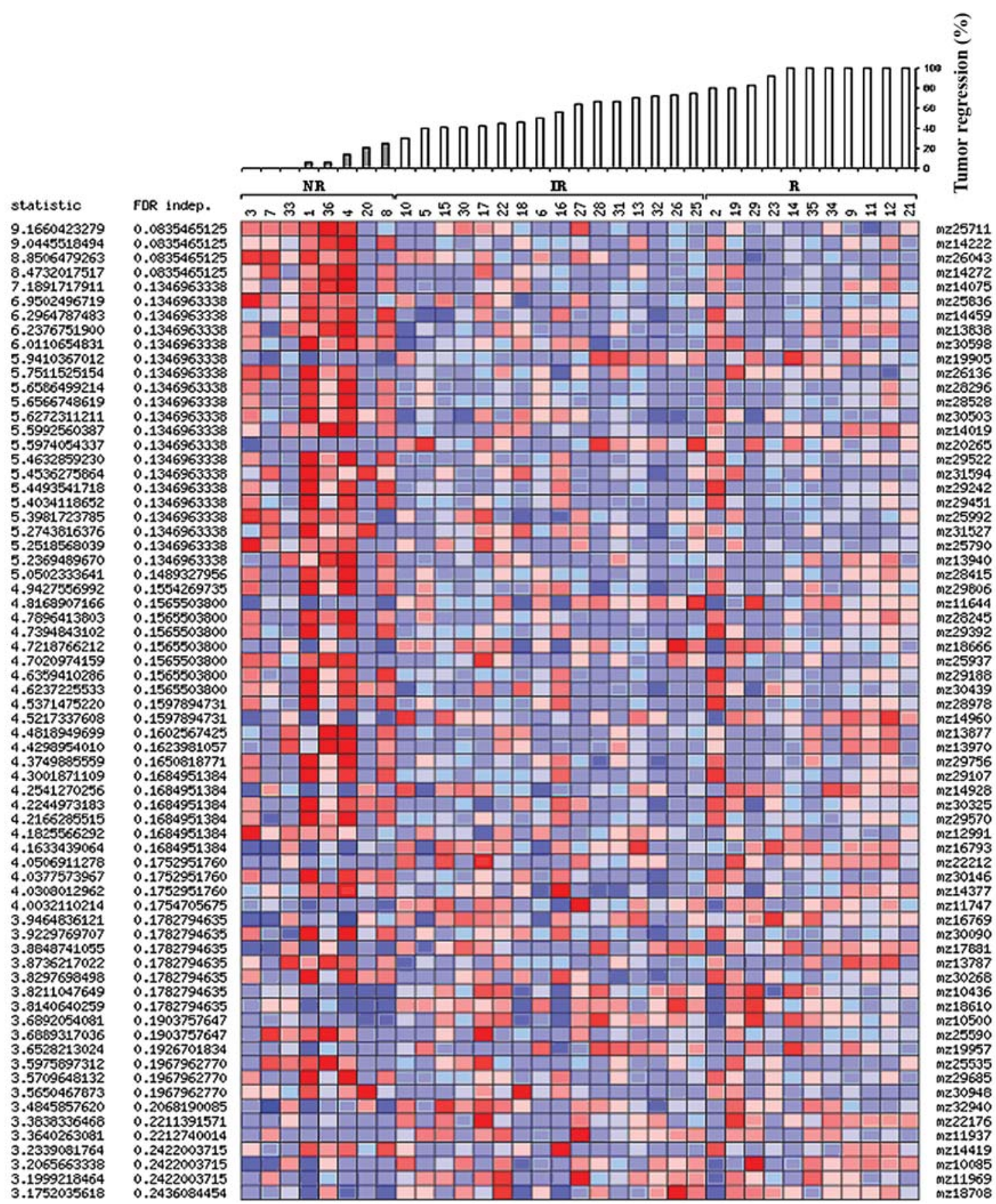

Figure 3. Heat map display of $\mathrm{m} / \mathrm{z}$ signals with significant differences among NR, IR and $\mathrm{R}$ groups in the training data set ( $\mathrm{n}=35$ ). The 35 cases in the training set were classified into three groups based on tumor regression rate (TRR): NR group (TRR $\leq 25 \%), 8$ cases; IR group ( $25 \%<\mathrm{TRR} \leq 75 \%$ ), 16 cases; R group (TRR $>75 \%$ ), 11 cases. Normalized $\mathrm{m} / \mathrm{z}$ values were compared by multi-class ANOVA statistics using the T-REX tool set of the GEPAS software. Each column represents a case as labeled on top, and each row represents a normalized $\mathrm{m} / \mathrm{z}$ signal as indicated at the right. ANOVA statistics and adjusted p-values using the FDR procedure of Benjamini and Hochberg are presented at the left. Sixty-eight peaks with FDR indep. $<0.25$ are displayed.

The subset of peaks meeting the median 2-fold difference, and $\mathrm{p}<0.05$ by Wilcoxon rank sum test criteria, were then used to build a classification rule. The rule was built using four different supervised classification algorithms in the GEPAS software: (i) Diagonal Linear Discriminant Analysis (DLDA), (ii) K-Nearest Neighbor Clustering (KNN), (iii) Support Vector Machines (SVM), (iv) Prediction Analysis with Microarrays (PAM). The accuracy for each of these methods during predictor building was estimated by the leave-one-out permutation method in the training set. In the validation data set, the method with the highest accuracy was selected as the optimal predicting algorithm. In both the univariate and the multivariate analyses, accuracy was defined as the percent of a given group that was correctly classified. Overall (unweighted) accuracy was defined as the average accuracy across all groups.

\section{Results}

Clinical response of breast tumors to neoadjuvant chemotherapy. The characteristics of the 52 patients and their tumor histopathologic features are summarized in Table I. The 
Table II. Differential $\mathrm{m} / \mathrm{z}$ signals separating drug resistant $(\mathrm{NR}, \mathrm{n}=8)$ from more susceptible breast cancers $(\mathrm{ST}, \mathrm{n}=27)$ in training set.

\begin{tabular}{|c|c|c|c|c|}
\hline Signal & NR median & ST Median & NR/ST & Wilcoxon p-value \\
\hline $\mathrm{m} / \mathrm{z} 10085$ & 13.552 & 55.207 & 0.245 & 0.010 \\
\hline $\mathrm{m} / \mathrm{z} 10290$ & 15.934 & 37.951 & 0.420 & 0.025 \\
\hline $\mathrm{m} / \mathrm{z} 10318$ & 11.658 & 40.645 & 0.287 & 0.007 \\
\hline $\mathrm{m} / \mathrm{z} 10373$ & 11.248 & 24.366 & 0.462 & 0.031 \\
\hline $\mathrm{m} / \mathrm{z} 10436$ & 8.459 & 19.550 & 0.433 & 0.012 \\
\hline $\mathrm{m} / \mathrm{z} 10500$ & 5.629 & 17.697 & 0.318 & 0.005 \\
\hline $\mathrm{m} / \mathrm{z} 10542$ & 4.322 & 11.621 & 0.372 & 0.045 \\
\hline $\mathrm{m} / \mathrm{z} 10821$ & 7.045 & 20.499 & 0.344 & 0.005 \\
\hline $\mathrm{m} / \mathrm{z} 10841$ & 5.031 & 29.447 & 0.171 & 0.003 \\
\hline $\mathrm{m} / \mathrm{z} 11124$ & 4.413 & 10.914 & 0.404 & 0.018 \\
\hline $\mathrm{m} / \mathrm{z} 11171$ & 5.621 & 16.857 & 0.333 & 0.020 \\
\hline m/z 11197 & 5.427 & 17.027 & 0.319 & 0.041 \\
\hline m/z 11370 & 5.025 & 11.535 & 0.436 & 0.045 \\
\hline m/z 11644 & 13.724 & 42.135 & 0.326 & 0.003 \\
\hline m/z 11717 & 18.920 & 41.174 & 0.460 & 0.008 \\
\hline m/z 11747 & 14.819 & 48.286 & 0.307 & 0.003 \\
\hline m/z 11937 & 5.626 & 13.663 & 0.412 & 0.013 \\
\hline m/z 11969 & 6.103 & 13.868 & 0.440 & 0.020 \\
\hline m/z 12991 & 6.375 & 3.114 & 2.047 & 0.028 \\
\hline $\mathrm{m} / \mathrm{z} 13838$ & 36.200 & 13.824 & 2.619 & 0.045 \\
\hline m/z 14019 & 22.488 & 7.148 & 3.146 & 0.050 \\
\hline $\mathrm{m} / \mathrm{z} 14075$ & 22.362 & 6.792 & 3.293 & 0.050 \\
\hline $\mathrm{m} / \mathrm{z} 14222$ & 10.193 & 3.486 & 2.924 & 0.005 \\
\hline $\mathrm{m} / \mathrm{z} 14272$ & 10.680 & 2.602 & 4.104 & 0.006 \\
\hline m/z 14377 & 7.356 & 3.533 & 2.082 & 0.020 \\
\hline $\mathrm{m} / \mathrm{z} 14459$ & 9.953 & 4.700 & 2.118 & 0.013 \\
\hline $\mathrm{m} / \mathrm{z} 14960$ & 19.682 & 48.587 & 0.405 & 0.017 \\
\hline m/z 16793 & 23.311 & 46.710 & 0.499 & 0.020 \\
\hline m/z 17881 & 3.785 & 42.412 & 0.089 & 0.007 \\
\hline m/z 18391 & 10.419 & 22.691 & 0.459 & 0.008 \\
\hline $\mathrm{m} / \mathrm{z} 18610$ & 7.293 & 14.585 & 0.500 & 0.010 \\
\hline $\mathrm{m} / \mathrm{z} 18666$ & 5.670 & 14.485 & 0.391 & 0.005 \\
\hline $\mathrm{m} / \mathrm{z} 18708$ & 5.884 & 13.414 & 0.439 & 0.015 \\
\hline m/z 19744 & 2.749 & 6.478 & 0.424 & 0.031 \\
\hline m/z 19905 & 2.344 & 12.108 & 0.194 & 0.000 \\
\hline m/z 19957 & 4.267 & 9.181 & 0.465 & 0.004 \\
\hline $\mathrm{m} / \mathrm{z} 20098$ & 3.608 & 7.559 & 0.477 & 0.045 \\
\hline $\mathrm{m} / \mathrm{z} 20186$ & 2.459 & 5.287 & 0.465 & 0.006 \\
\hline $\mathrm{m} / \mathrm{z} 20265$ & 1.640 & 5.828 & 0.281 & 0.000 \\
\hline $\mathrm{m} / \mathrm{z} 20434$ & 0.977 & 2.859 & 0.342 & 0.028 \\
\hline $\mathrm{m} / \mathrm{z} 20663$ & 2.169 & 5.907 & 0.367 & 0.028 \\
\hline $\mathrm{m} / \mathrm{z} 22176$ & 3.542 & 16.807 & 0.211 & 0.003 \\
\hline $\mathrm{m} / \mathrm{z} 22212$ & 2.852 & 13.960 & 0.204 & 0.002 \\
\hline $\mathrm{m} / \mathrm{z} 25711$ & 3.363 & 1.097 & 3.066 & 0.002 \\
\hline $\mathrm{m} / \mathrm{z} 25790$ & 4.608 & 1.803 & 2.556 & 0.041 \\
\hline $\mathrm{m} / \mathrm{z} 25836$ & 4.815 & 1.687 & 2.854 & 0.007 \\
\hline $\mathrm{m} / \mathrm{z} 25937$ & 4.044 & 1.288 & 3.139 & 0.041 \\
\hline $\mathrm{m} / \mathrm{z} 25992$ & 4.333 & 2.003 & 2.163 & 0.041 \\
\hline
\end{tabular}


Table II. Continued.

\begin{tabular}{|c|c|c|c|c|}
\hline Signal & NR median & ST Median & NR/ST & Wilcoxon p-value \\
\hline $\mathrm{m} / \mathrm{z} 26043$ & 4.137 & 1.602 & 2.583 & 0.034 \\
\hline $\mathrm{m} / \mathrm{z} 26136$ & 2.620 & 1.063 & 2.464 & 0.031 \\
\hline $\mathrm{m} / \mathrm{z} 28978$ & 18.523 & 7.817 & 2.369 & 0.037 \\
\hline $\mathrm{m} / \mathrm{z} 30598$ & 3.364 & 1.663 & 2.023 & 0.018 \\
\hline $\mathrm{m} / \mathrm{z} 30892$ & 2.196 & 1.044 & 2.104 & 0.020 \\
\hline $\mathrm{m} / \mathrm{z} 30948$ & 1.905 & 0.800 & 2.381 & 0.006 \\
\hline $\mathrm{m} / \mathrm{z} 31594$ & 2.427 & 1.144 & 2.121 & 0.025 \\
\hline $\mathrm{m} / \mathrm{z} 31848$ & 1.956 & 0.713 & 2.744 & 0.041 \\
\hline
\end{tabular}

The $\mathrm{m} / \mathrm{z}$ signals were selected by both the median-fold difference (minimally 2 -fold) and statistical significance (Wilcoxon test, $\mathrm{p}<0.05$ ). This produced a set of 56 signals from a total of 300 signals.

average age of the patients was 49.4 (range 29-84) years. After neoadjuvant chemotherapy, six patients had no evidence of residual invasive or in situ disease at the primary tumor site, and no metastasis in lymph nodes; two patients had residual ductal carcinoma in situ (DCIS) and positive lymph nodes; one patient had no residual invasive or in situ cancer in the breast but still had micrometastasis $(0.05 \mathrm{~mm})$ in a single node; and 43 patients had residual invasive cancers with $62.8 \%$ (27/43) having positive lymph nodes. Before chemotherapy the mean tumor sizes for the 9 pathological complete tumor response (pCR) and 43 non-pCR patients were 7.17 and $8.40 \mathrm{~cm}$, respectively. Of the 43 non-pCR patients, 12 were classified as drug resistant with a calculated TRR $\leq 25 \%$ who were also considered as non-responders (NR). Eight of $12 \mathrm{NR}$ were used in the training dataset and 4 in the validation dataset.

Comparison of SELDI spectra from non-responders (NR) and chemo-susceptible tumors $(S T)$. The median intensity SELDI-TOF spectra of the NR and ST groups from the total patient cohort are shown in Fig. 1. Fifty-nine of a total of $300 \mathrm{~m} / \mathrm{z}$ peaks were found to have significantly different median peak intensity in the $S T(n=40)$ vs. NR $(n=12)$ groups using the criterion $\mathrm{p}<0.05$ (data not shown). In particular, the difference in peak intensity at $\mathrm{m} / \mathrm{z} 20,265$ correctly classified $91.7 \%(11 / 12)$ of the NR tumors and $75 \%$ (30/40) of the ST tumors with an overall unweighted accuracy of $82.5 \%$ $(\mathrm{p}=0.0014)$.

Comparison of SELDI spectra from non-responders (NR) and pathologically complete responders $(p C R)$. The median intensity SELDI-TOF spectra of the NR and pCR groups are shown in Fig. 2. Thirty-one of a total of $198 \mathrm{~m} / \mathrm{z}$ peaks were found to have significantly different median peak intensity in the NR $(n=12)$ vs. pCR $(n=9)$ groups using the criterion $\mathrm{p}<0.05$ (data not shown). In particular, the median intensity of the $\mathrm{m} / \mathrm{z} 16,905$ peak was 30.6 in the NR group and 17.1 in the pCR group. With a separating threshold intensity of 20.4 , 11 of 12 patients with NR tumors were above, and 8 of 9 pCR tumors were below the threshold. Thus $88.9 \%$ of the pCR tumors and $91.7 \%$ of the NR tumors were correctly classified
Table III. Comparison of the accuracy of four different methods used for building the unbiased predictor by permutation tests (the leave-one-out approach) in the training set.

\begin{tabular}{lcccc}
\hline & DLDA & KNN & SVM & PAM \\
\hline Accuracy (\%) & 85.7 & 76.6 & 69.3 & 80.0 \\
\hline
\end{tabular}

The methods used for building the unbiased predictor include (A) Diagonal Linear Discriminant Analysis (DLDA), (B) Nearest neighbor (KNN), (C) Support Vector Machines (SVM) and (D) Prediction Analysis with Microarrays (PAM). The cross-validation accuracy for the selected set of signals were tested by the leave-oneout approach in the training set, and the DLDA method was found to have the highest accuracy $(85.7 \%)$.

by the difference of the peak intensity at $\mathrm{m} / \mathrm{z} 16905$. The overall unweighted accuracy was $90.3 \%$.

Proteomic pattern of tumor responses to neoadjuvant chemotherapy in the training set. A three-group comparison was first performed in the training set to explore the spectral differences between the NR, IR and R groups by ANOVA using the T-REX toolset of the GEPAS software. A set of 68 peaks was identified with significant changes across the NR, IR and $\mathrm{R}$ groups (FDR-indep. $\mathrm{p}<0.25$, Fig. 3). Noticeably, more prominent and significant changes appeared between NR and the other two $(\mathrm{R}+\mathrm{IR})$ groups. Therefore, in the subsequent comparison and in the validation set, the $\mathrm{R}$ and IR groups were collapsed into one 'susceptible' (ST) group and compared to the 'resistant' (NR) group.

From the initial 300 peaks, 56 were identified as being different between NR and ST tumors in the training set as defined by the criteria of at least a 2-fold difference of peak intensity, and statistical significance of $\mathrm{p}<0.05$ by Wilcoxon test (Table II). This set of 56 peaks was employed to build a classifier using four classification algorithms in the GEPAS (DLDA, KNN, SVM and PAM) to predict tumor response in the validation set. In the training set, the performance of 
Table IV. Performance comparison of various supervised classifications of tumor response to neoadjuvant chemotherapy in the validation set.

\begin{tabular}{lccc}
\hline & $\begin{array}{c}\text { Percentage of correctly } \\
\text { predicted in NR (\%) }\end{array}$ & $\begin{array}{c}\text { Percentage of correctly } \\
\text { predicted in ST (\%) }\end{array}$ & $\begin{array}{c}\text { Overall } \\
\text { accuracy }(\%)\end{array}$ \\
\hline DLDA & $100(4 / 4)$ & $0.0(0 / 13)$ & 50.0 \\
KNN & $100(4 / 4)$ & $84.6(11 / 13)$ & 92.3 \\
SVM & $0(0 / 4)$ & $92.3(12 / 13)$ & 46.2 \\
PAM & $25(1 / 4)$ & $84.6(11 / 13)$ & 54.8 \\
\hline
\end{tabular}

The predictors derived from the training set were used to predict chemo-responses in the validation set $(n=17)$. The performances of the four algorithms (DLDA, KNN, SVM and PAM) were tested and compared. Accuracy rates of these methods ranged 46.2-92.3\%. The best prediction was obtained by the KNN model where $\mathrm{K}=1$. The KNN model correctly classified $100 \%$ of resistant tumors and $84.6 \%$ of the tumors with favorable response, achieving an overall accuracy rate of $92.3 \%$.

these algorithms was evaluated by cross-validation using a permutation test (leave-one-out approach). As shown in Table III, the accuracy rates of these methods were similar (69.3-85.7\%) with the highest accuracy achieved by the DLDA method.

Predicting breast tumor responses to neoadjuvant chemotherapy in the validation set. The 56 peaks (predictors) derived from the training set were then used to classify the 17 cases in the validation data set and predict the chemoresponses. The performances of the four algorithms (DLDA, KNN, SVM and PAM) were tested and compared (Table IV). Accuracy rates of these methods were between 46.2-92.3\%. The optimal prediction was obtained by the $\mathrm{KNN}$ model where $\mathrm{K}=1$. The KNN model correctly classified $100 \%$ of resistant tumors $(4 / 4)$ and $84.6 \%$ of the susceptible tumors (11/13), achieving an overall accuracy rate of $92.3 \%$.

\section{Discussion}

In this study, SELDI-TOF mass spectrometry was used to compare the protein profiles of 52 breast tumor specimens derived from patients with large breast cancers who had known responses to a defined neoadjuvant regimen. To our knowledge, this is the first attempt to use MS generated profiles to predict the response of human breast cancer tissue to chemotherapy. The proteomic differences in groups of patients with either susceptible or resistant tumors to neoadjuvant chemotherapy were investigated, and differential proteomic signals were selected. These candidate signals appeared to correlate well with clinical response of the tumors to the neoadjuvant chemotherapy. Using the methods described, we found that many prominent proteomic differences existed between drug resistant breast tumors and those with various degrees of drug response. This result suggests the presence of a unique proteomic signature characteristic of tumor chemosensitivity.

Even with these highly selective analytical tools, it was not possible to accurately predict tumor responses to treatment in all cases. Two of the 13 chemotherapy susceptible tumors were misclassified as resistant tumors. The two misclassified cases were $\mathrm{T} 3$ and $\mathrm{T} 4$ infiltrating ductal carcinomas respectively, with one being a triple negative tumor (negative for estrogen receptor, progesterone receptor and HER2/neu), and the other being progesterone receptor negative and HER2/neu negative. From a clinical point of view, it is possible that triple negative or negative estrogen and/or progesterone receptors are sufficient to predict a favorable response to taxotere and carboplatin (21). Our studies suggest that both proteomic biomarkers and clinical variables should be taken into account in building future prediction models.

When the four algorithms were compared in the training set, DLDA was found to have the highest accuracy (85.7\%) by permutation test. However in the validation set, KNN was shown to have the highest accuracy $(92.3 \%)$. The inconsistency of this finding derived from the training and validation data sets may be due to the small sample sizes. Also with the limited sample size especially in the validation group, the accuracy in the DLDA and SVM methods are low. DLDA did predict 4 out of 4 NR, but failed to predict ST. Meanwhile, SVM predicted 12 out of $13 \mathrm{ST}$ but missed the NR. A larger sample size may improve the consistency and accuracy in building and testing the prediction model.

Several types of proteomic analytical tools are being used to study disease-related biomarkers. These include the openended technologies such as 2-dimension gel electrophoresis and mass spectrometry, and closed-ended technologies such as antibody microarrays, antibody bead arrays, reverse phase protein microarrays, and tissue microarrays. Mass spectrometry-based technologies are being continually refined for proteomic profiling since their introduction into this field about a decade ago. SELDI-TOF and MALDI-TOF have been used to profile serum and plasma with the aim of identifying proteomic fingerprints that may differentiate individuals with normal breasts from cancerous breasts (22-26), predict in vitro response of breast cancer cell lines to chemotherapy $(27,28)$, and predict the clinical tumor response to chemotherapy using plasma samples (29).

However, in recent years SELDI/MALDI based proteomic profiling has faced criticisms concerning the sensitivity, reproducibility and inability to identify candidate proteins with most criticisms directed to serum profiling studies (30-35). Cancer related proteins when present in serum are trace in amount because they are diluted in the large volume of blood, 
and masked by numerous other abundant proteins that normally occur in the circulation. These problems are either not relevant or are less important for the direct analysis of tumor tissue, as performed in this study. Standardized protocols were used for tissue collection, storage and sample preparation to minimize variability between samples. We have also previously demonstrated that the SELDI spectra of the same specimens are relatively stable and reproducible, with virtually identical spectra produced from the same sample upon re-analysis several days later (36). Since the SELDI-TOF technology enables a straightforward analysis of protein biomarkers, it provides a means to rapidly evaluate intact protein patterns in a large number of specimens. It was therefore chosen as the first attempt to profile breast cancer tissues with different responses to neoadjuvant chemotherapy.

Our results show that SELDI-TOF MS protein profiling of breast cancer tissue obtained before chemotherapy may predict the chemoresponse of breast cancer. There were many prominent proteomic differences between drug resistant breast tumors and those with various degrees of drug response. The major disadvantage of SELDI/MALDI is the inability of direct identification of promising biomarker signals by the technology. Our future study will concentrate on extending these findings with larger sample sizes, and simultaneously identifying the proteins that generate the SELDI signals of interest using nano-LC-Orbitrap instrumentation from shotgun digests of the same samples.

\section{Acknowledgements}

We thank Jeffrey A. Gornbein (Department of Biomathematics, UCLA) for providing assistance for the SELDI date processing and critical revision advice on statistical analysis. The present study was supported by grant funds from NIH (NCI \#1RO1 CA 093736-01A1), the Gonda Foundation, Entertainment Industry Foundation (EIF)/ Women's Cancer Research Fund, and Friends of the Breast Program at UCLA.

\section{References}

1. Hanrahan EO, Hennessy BT and Valero V: Neoadjuvant systemic therapy for breast cancer: an overview and review of recent clinical trials. Expert Opin Pharmacother 6: 1477-1491, 2005.

2. Kaufmann M, von Minckwitz G and Rody A: Preoperative (neoadjuvant) systemic treatment of breast cancer. Breast 14: 576-581, 2005.

3. Goldhirsch A, Glick JH, Gelber RD, Coates AS, Thürlimann B and Senn HJ: Meeting highlights: international expert consensus on the primary therapy of early breast cancer 2005. Ann Oncol 16: 1569-1583, 2005.

4. Carlson RW, Brown E, Burstein HJ, et al: NCCN task force report: adjuvant therapy for breast cancer. J Natl Compr Cancer Netw 4: S1-S26, 2006.

5. Cianfrocca $\mathrm{M}$ and Wolff AC: Optimizing endocrine therapy for premenopausal and postmenopausal women with breast cancer. Oncology 21: 63-69, 2007.

6. Prowell TM and Davidson NE: What is the role of ovarian ablation in the management of primary and metastatic breast cancer today? Oncologist 9: 507-517, 2004.

7. Early Breast Cancer Trialists' Collaborative Group: Ovarian ablation in early breast cancer: overview of the randomised trials. Lancet 348: 1189-1196, 1996.

8. Gibson L, Dawson C, Lawrence D and Bliss J: Aromatase inhibitors for treatment of advanced breast cancer in postmenopausal women. Cochrane Database Syst Rev 1: CD003370, DOI: 10.1002/14651858.CD003370.pub2, 2007.
9. Carlson RW, Hudis CA, Pritchard KI, et al: Adjuvant endocrine therapy in hormone receptor-positive postmenopausal breast cancer: evolution of NCCN, ASCO, and St Gallen recommendations. J Natl Compr Cancer Netw 4: 971-979, 2006.

10. Romond EH, Perez EA, Bryant J, et al: Trastuzumab plus adjuvant chemotherapy for operable HER2-positive breast cancer. N Engl J Med 353: 1673-1684, 2005.

11. Piccart-Gebhart MJ, Procter M, Leyland-Jones B, et al: Trastuzumab after adjuvant chemotherapy in HER2-positive breast cancer. N Engl J Med 353: 1659-1672, 2005.

12. Pegram MD, Pauletti G and Slamon DJ: HER-2/neu as a predictive marker of response to breast cancer therapy. Breast Cancer Res Treat 52: 65-77, 1998.

13. Paik S, Tang G, Shak S, et al: Gene expression and benefit of chemotherapy in women with node-negative, estrogen receptorpositive breast cancer. J Clin Oncol 24: 3726-3734, 2006.

14. Paik S, Shak S, Tang G, et al: A multigene assay to predict recurrence of tamoxifen-treated, node-negative breast cancer. $\mathrm{N}$ Engl J Med 351: 2817-2826, 2004.

15. Acharya CR, Hsu DS, Anders CK, et al: Gene expression signatures, clinicopathological features, and individualized therapy in breast cancer. JAMA 299: 1574-1587, 2008.

16. Somiari RI, Somiari S, Russell S and Shriver CD: Proteomics of breast carcinoma. J Chromatogr B Analyt Technol Biomed Life Sci 815: 215-225, 2005.

17. Somiari RI, Sullivan A, Russell S, et al: High-throughput proteomic analysis of human infiltrating ductal carcinoma of the breast. Proteomics 3: 1863-1873, 2003.

18. Li X, Gentleman R, Lu X, Shi Q, Lglehart JD, Harris L and Miron A: SELDI-TOF Mass Spectrometry Protein Data. Bioinformatics and Computational Biology Solutions using $\mathrm{R}$ and Bioconductor, Springer, Chapter 6: 91-109, 2005.

19. Shen D, Chang HR, Chen Z, et al: Loss of annexin A1 expression in human breast cancer detected by multiple high-throughput analyses. Biochem Biophys Res Commun 326: 218-227, 2005.

20. Shen D, Nooraie F, Elshimali Y, et al: Decreased expression of annexin A1 is correlated with breast cancer development and progression as determined by a tissue microarray analysis. Hum Pathol 37: 1583-1591, 2006.

21. Chang HR, Slamon D, Gornbein JA and Chung D: Preferential pathologic complete response (pCR) by triple-negative (-) breast cancer to neoadjuvant docetaxel (T) and carboplatin (C). J Clin Oncol 26: (May 20 suppl), 604, 2008.

22. Laronga $C$ and Drake RR: Proteomic approach to breast cancer. Cancer Control 14: 360-368, 2007.

23. Hu Y, Zhang S, Yu J, Liu J and Zheng S: SELDI-TOF-MS: The proteomics and bioinformatics approaches in the diagnosis of breast cancer. Breast 14: 250-255, 2005.

24. Li J, Zhang Z, Rosenzweig J, Wang YY and Chan DW: Proteomics and bioinformatics approaches for identification of serum biomarkers to detect breast cancer. Clin Chem 48: 1296-1304, 2002.

25. Hudelist G, Singer CF, Pischinger KI, Kaserer K, Manavi M, Kubista E and Czerwenka KF: Proteomic analysis in human breast cancer: Identification of a characteristic protein expression profile of malignant breast epithelium. Proteomics 6: 1989-2002, 2006.

26. Dwek MV and Alaiya AA: Proteome analysis enables separate clustering of normal breast, benign breast and breast cancer tissues. Br J Cancer 89: 305-307, 2003.

27. Mian S, Ball G, Hornbuckle J, et al: A prototype methodology combining surface-enhanced laser desorption/ionization protein chip technology and artificial neural network algorithms to predict the chemoresponsiveness of breast cancer cell lines exposed to Paclitaxel and Doxorubicin under in vitro conditions. Proteomics 3: 1725-1737, 2003.

28. Chuthapisith S, Layfield R, Kerr ID, Hughes C and Eremin O: Proteomic profiling of MCF-7 breast cancer cells with chemoresistance to different types of anti-cancer drugs. Int J Oncol 30: 1545-1551, 2007

29. Pusztai L, Gregory BW, Baggerly KA, et al: Pharmacoproteomic analysis of prechemotherapy and postchemotherapy plasma samples from patients receiving neoadjuvant or adjuvant chemotherapy for breast carcinoma. Cancer 100: 1814-1822, 2004.

30. Diamandis EP: Mass spectrometry as a diagnostic and a cancer biomarker discovery tool: opportunities and potential limitations. Mol Cell Proteomics 3: 367-378, 2004.

31. Diamandis EP: Analysis of serum proteomic patterns for early cancer diagnosis: drawing attention to potential problems. J Natl Cancer Inst 96: 353-356, 2004. 
32. Ransohoff DF: Lessons from controversy: ovarian cancer screening and serum proteomics. J Natl Cancer Inst 97: 315-319, 2005 .

33. Liotta LA, Lowenthal M, Mehta A, Conrads TP, Veenstra TD Fishman DA and Petricoin EF III: Importance of communication between producers and consumers of publicly available experimental data. J Natl Cancer Inst 97: 310-314, 2005.

34. Diamandis EP: Serum proteomic profiling by matrix-assisted laser desorption-ionization time-of-flight mass spectrometry for cancer diagnosis: next steps. Cancer Res 66: 5540-5541, 2006
35. Van der Merwe DE, Oikonomopoulou K, Marshall J and Diamandis EP: Mass Spectrometry: uncovering the cancer proteome for diagnostics. Adv Cancer Res 96: 23-50, 2007.

36. He J, Gornbein J, Shen D, et al: Detection of breast cancer biomarkers in nipple aspirate fluid by SELDI-TOF and their identification by combined liquid chromatography-tandem mass spectrometry. Int J Oncol 30: 145-154, 2007. 